\title{
Volume of $\beta$-Bursts, But Not Their Rate, Predicts Successful Response Inhibition
}

\author{
${ }^{\circledR}$ Nadja Enz, ${ }^{1}{ }^{\circledR}$ Kathy L. Ruddy, ${ }^{1}{ }^{\circledR}$ Laura M. Rueda-Delgado, ${ }^{1}$ and Robert Whelan ${ }^{1,2}$ \\ ${ }^{1}$ School of Psychology and Institute of Neuroscience, Trinity College Dublin, Dublin, D02 PN40, Ireland, and ${ }^{2}$ Global Brain Health Institute, Trinity \\ College Dublin, Dublin, D02 PN40, Ireland
}

In humans, impaired response inhibition is characteristic of a wide range of psychiatric diseases and of normal aging. It is hypothesized that the right inferior frontal cortex (rIFC) plays a key role by inhibiting the motor cortex via the basal ganglia. The electroencephalography (EEG)-derived $\beta$-rhythm $(15-29 \mathrm{~Hz})$ is thought to reflect communication within this network, with increased right frontal $\beta$-power often observed before successful response inhibition. Recent literature suggests that averaging spectral power obscures the transient, burst-like nature of $\boldsymbol{\beta}$-activity. There is evidence that the rate of $\boldsymbol{\beta}$-bursts following a Stop signal is higher when a motor response is successfully inhibited. However, other characteristics of $\boldsymbol{\beta}$-burst events, and their topographical properties, have not yet been examined. Here, we used a large human (male and female) EEG Stop Signal task (SST) dataset $(n=218)$ to examine averaged normalized $\beta$-power, $\beta$-burst rate, and $\beta$-burst "volume" (which we defined as burst duration $x$ frequency span $\times$ amplitude). We first sought to optimize the $\beta$-burst detection method. In order to find predictors across the whole scalp, and with high temporal precision, we then used machine learning to (1) classify successful versus failed stopping and to (2) predict individual stop signal reaction time (SSRT). $\beta$-burst volume was significantly more predictive of successful and fast stopping than $\beta$-burst rate and normalized $\beta$-power. The classification model generalized to an external dataset $(n=201)$. We suggest $\beta$-burst volume is a sensitive and reliable measure for investigation of human response inhibition.

Key words: $\beta$-bursts; $\beta$-oscillations; electroencephalography; human; response inhibition; stop signal task

Significance Statement

The electroencephalography (EEG)-derived $\beta$-rhythm $(15-29 \mathrm{~Hz})$ is associated with the ability to inhibit ongoing actions. In this study, we sought to identify the specific characteristics of $\beta$-activity that contribute to successful and fast inhibition. In order to search for the most relevant features of $\beta$-activity, across the whole scalp and with high temporal precision, we employed machine learning on two large datasets. Spatial and temporal features of $\beta$-burst "volume" (duration $\times$ frequency span $\times$ amplitude) predicted response inhibition outcomes in our data significantly better than $\beta$-burst rate and normalized $\beta$-power. These findings suggest that multidimensional measures of $\beta$-bursts, such as burst volume, can add to our understanding of human response inhibition.

\footnotetext{
Received Aug. 25, 2020; revised Mar. 10, 2021; accepted Mar. 11, 2021.

Author contributions: N.E., K.L.R., L.M.R.-D., and R.W. designed research; N.E., K.L.R., L.M.R.-D., and R.W. performed research; N.E., K.L.R., L.M.R.-D., and R.W. analyzed data; N.E., K.L.R., L.M.R.-D., and R.W. wrote the paper.

This cluster was funded through grants from Science Foundation Ireland. N.E. was supported by the Irish Research Council Postgraduate Scholarship GOIPG/2018/537. K.L.R. was supported by the Irish Research Council Grant G0IPD/2017/798 and the Health Research Board, Ireland Grant HRB-EIA-2019-003. L.M.R.-D. was supported by the Science Foundation Ireland Grant SFI 18/IF/6272. R.W. was supported by the Science Foundation Ireland Grant 16/ERCD/3797, the European Foundation for Alcohol Research (ERAB), the Brain \& Behavior Research Foundation Grant 23599, and the Health Research Board Grant HRAPOR-2015-1075. We thank Dr. Jan Wessel for sharing his Stop Signal Task electroencephalography data and for his advice in interpreting the data and analysis scripts. We also thank Dr. Hanni Kiiski, Dr. Laura O'Halloran, Rory Boyle, Laura Rai, and Gabi Pragulbickaite for help with data collection and electroencephalography quality control. All machine learning calculations were performed on the Lonsdale cluster maintained by the Trinity Centre for High Performance Computing.

The authors declare no competing financial interests.

Correspondence should be addressed to Robert Whelan at robert.whelan@tcd.ie.

https://doi.org/10.1523/JNEUROSCI.2231-20.2021

Copyright $\odot 2021$ the authors
}

\section{Introduction}

The ability to inhibit unwanted or inappropriate behaviors relies on effective response inhibition in the brain. The Stop Signal task (SST) measures this cognitive process (Logan and Cowan, 1984) by requiring the participant to cancel an already initiated motor response following an infrequent Stop cue. If the response is withheld following the Stop cue then the trial is classified as a "success." If a response is made then the trial is classified as a "failure." The stop signal reaction time (SSRT) is an estimation of the covert latency of the action cancellation process (Verbruggen et al., 2019). The SSRT is $\sim 200-250 \mathrm{~ms}$ in healthy adults, and slower SSRTs are characteristic of several psychiatric diseases (Lijffijt et al., 2005; Luijten et al., 2011) and normal aging (Hsieh and Lin, 2017).

Many previous studies have suggested that right inferior frontal cortex (rIFC) is involved in response inhibition through a 
rIFC-basal ganglia-motor cortex pathway (Aron et al., 2014; Wessel and Aron, 2017). Jana et al. (2020) recently supported the role of this pathway in action stopping by suggesting an exact temporal cascade: rIFC activation at $120 \mathrm{~ms}$ after Stop signal, global motor suppression at $140 \mathrm{~ms}$, muscle inhibition at $160 \mathrm{~ms}$, and SSRT at $220 \mathrm{~ms}$. Communication in this pathway may be facilitated through brain oscillations in the $\beta$-frequency band (15-29 Hz), and several studies have reported an increase in averaged $\beta$-power over the rIFC at the moment of stopping a movement (Swann et al., 2009; Wagner et al., 2018; Schaum et al., 2020).

Recent literature suggests that the cortical $\beta$-rhythm is by nature characterized by short-lasting, transient bursts and only appears to change in sustained amplitude if it is averaged over multiple trials (Feingold et al., 2015; Sherman et al., 2016). Various characteristics of $\beta$-bursts have been analyzed in previous studies, such as rate, timing, probability, peak power, duration, and interval time (Feingold et al., 2015; Sherman et al., 2016; Shin et al., 2017; Tinkhauser et al., 2017; Errington et al., 2020; Hannah et al., 2020; Jana et al., 2020; Seedat et al., 2020; Wessel, 2020). Three recent studies have investigated the role of $\beta$-bursts in human response inhibition using electroencephalography (EEG) and have reported mixed findings. Wessel (2020) found a larger frontocentral $\beta$-burst rate for successful compared with failed action stopping whereas Jana et al. (2020) did not find a significant difference. Another study reported that earlier right frontal $\beta$-bursts were associated with faster CancelTime, a measure of stopping latency using electromyography (EMG; Hannah et al., 2020).

Here, we aimed to further quantify the role and nature of $\beta$-bursts in human response inhibition in two large SST EEG datasets $(n=419)$ by using machine learning to search a wide range of spatial and temporal features. First, we sought to optimize the $\beta$-burst detection method. In a second step, we conducted two different single-trial analyses to (1) classify successful versus failed Stop trials and (2) predict an individual's SSRT. We investigated two $\beta$-burst characteristics: rate and volume (a composite measure of burst duration, frequency span and amplitude) and also compared them to averaged normalized $\beta$-power. Given the high dimensional nature of the data, we employed a machine-learning approach, employing best-practice for quantifying the generalizability of our results (Poldrack et al., 2020). We trained the models on $60 \%$ of our data (internal validation set; $n=130$ ) and subsequently applied the resulting models on the remaining, unseen $40 \%$ of our data (holdout validation set; $n=88)$. We then aimed to validate results on an external dataset ( $n=201$; Wessel, 2020). We hypothesized that $\beta$-burst features will be more predictive than averaged normalized $\beta$-power for classifying successful versus failed Stop trials, and for predicting individual SSRTs. We also hypothesized that $\beta$-bursts over the right frontal scalp region would be most predictive.

\section{Materials and Methods}

\section{Participants}

The internal and holdout validation dataset consisted of 282 healthy adult human volunteers [age: $35.03 \pm 14.72$ years (mean \pm SD); 175 females] who were pooled from four studies conducted in University College Dublin and Trinity College Dublin, Ireland. The studies were approved by the ethics committees of University College Dublin School of Psychology and Trinity College Dublin School of Psychology. Participants provided written informed consent. The raw data from this dataset formed the basis of another study that did not focus on spectral properties (Rueda-Delgado et al., 2021). The inclusion and exclusion criteria of each project are available in Rueda-Delgado et al. (2021; their Supplementary Material).

The external validation dataset consisted of 214 healthy adult human volunteers [age: $22.51 \pm 6.67$ years (mean $\pm \mathrm{SD}$ ); 121 females]. This dataset was published as part of another study investigating $\beta$-bursts in response inhibition (Wessel, 2020) and is openly available on the Open Science Framework (https://osf.io/v3a78/).

Task

The task used for this study is described in detail in Rueda-Delgado et al. (2021). Briefly, participants underwent an adaptive visual SST. Each trial lasted $1000 \mathrm{~ms}$ and was preceded by a fixation cross (1000-ms duration). Participants were then presented with arrows pointing either to the right or left (Go stimulus) and they were instructed to respond with their right or left index finger, respectively, as fast as possible via an Xbox 360 game controller. In one of four Go trials, the Go stimulus was followed by an arrow pointing upwards (Stop signal) after a varying stop-signal delay (SSD). The participants were instructed to inhibit their button press on these Stop trials. The SSD was adjusted by a tracking algorithm, aiming to achieve a task difficulty resulting in $50 \%$ successful and $50 \%$ failed Stop trials. After a successful Stop trial, the SSD was increased, making the task harder and after a failed Stop trial, the SSD was decreased, making the task easier. The initial SSD was $250 \mathrm{~ms}$ and was subsequently adjusted using a double-limit algorithm (see Richards et al., 1999). The SSD could vary between 50 and $450 \mathrm{~ms}$. Following a Stop trial, the subsequent SSD value was chosen randomly between the current SSD and a pair of limits (higher or lower, as appropriate). These limits were designed to converge on the SSD that produced a $50 \%$ success rate and to be robust to fluctuations on individual trials. If a participant responded to the Go stimulus before Stop signal presentation, then the SSD was decreased for subsequent trials. The task consisted of 135 Go trials and 45 Stop trials and was presented in three blocks of 60 trials.

Participants in the external dataset performed a slightly different version of the SST which is described in Wessel (2020). The main differences were a higher Stop trial probability (0.33), a different visual Stop signal (arrow turned from white to red), different response buttons ( $\mathrm{q}$ and $\mathrm{p}$ buttons on a QWERTY keyboard), the tracking algorithm $( \pm 50 \mathrm{~ms})$ was implemented independently for leftward and rightward Go stimuli, and a larger number of trials (six blocks of 50 trials).

\section{SSRT analysis}

The SSRT was calculated using the integration method with replacement of Go omissions by the maximum reaction time (RT) (Verbruggen et al., 2019). All Go trials were included in the Go RT distribution, including Go trials with choice errors. Premature responses on failed Stop trials were included when calculating the probability of responding on a Stop trial $[\mathrm{p}$ (respond|signal)] and mean SSD. Participants with SSRT $<$ $125 \mathrm{~ms}$ and $>98$ th percentile of total SSRT distribution $(303.7 \mathrm{~ms})$ were excluded from the analysis $(n=47)$. The same criteria were applied to the external dataset [SSRT $<125 \mathrm{~ms}$ and $>98$ th percentile SSRT distribution $(385.7 \mathrm{~ms})$ ], and 11 participants were excluded.

\section{EEG recording and preprocessing}

64-Channel EEG data in the 10-5 system were recorded during the SST in a soundproof, darkened room using the ActiveTwo Biosemi system. Four additional electrodes recorded the electrooculogram from $\sim 2 \mathrm{~cm}$ below the eyes (vertical eye movements) and from the outer canthi (horizontal eye movements). Two further electrodes recorded from bilateral mastoids.

EEG data were digitized with a sampling rate of $512 \mathrm{~Hz}$. EEG data preprocessing was conducted using the EEGLAB toolbox (Delorme and Makeig, 2004; http://sccn.ucsd.edu/eeglab) in conjunction with the Fully Automated Statistical Thresholding for EEG artifact Rejection plug-in (FASTER; Nolan et al., 2010; http://sourceforge.net/projects/faster). The data were initially bandpass filtered between 0.1 and $95 \mathrm{~Hz}$, notch filtered at $50 \mathrm{~Hz}$ and average referenced across all scalp electrodes. Data were subsequently epoched from $500 \mathrm{~ms}$ before Go/Stop stimulus onset to $2000 \mathrm{~ms}$ after Go/Stop stimulus onset for Go trials and Stop trials, 
respectively. FASTER identified and removed artefactual (i.e., non-neural) independent components, removed epochs containing large artefacts (e.g., muscle twitches) and interpolated channels with poor signal quality. The remaining EEG data were then visually inspected by trained raters to ensure good quality and that any remaining noisy data were removed. Specifically, trained raters identified any remaining artefacts in independent components (e.g., eyeblinks), epochs containing idiosyncratic muscle/movement or transient electrode artifacts, high amount of $\alpha$-activity and interpolated any channels that were noisy throughout all epochs of a participant. Datasets with a large amount of artefacts (e.g., because of chewing) and/or with $>40 \%$ of epochs removed were excluded from further analysis. The remaining datasets were further checked for noisy data by calculating each dataset's event-related potential (ERP) and calculating $z$ scores across all ERPs. Datasets with $z$ scores $>15$ were excluded. After exclusion of these, new $z$ scores were calculated and datasets with $z$ scores $>3$ were excluded from further analysis. Fifteen participants were rejected from further analysis because of these artefacts or noisy data (in accordance with the exclusion criteria in Rueda-Delgado et al., 2021). The remaining 220 participants were subjected to further analysis. Details for EEG recording and preprocessing steps of the external dataset can be found in the original publication (Wessel, 2020). The external data were recorded using a different 62channel array. We therefore interpolated the channel locations from the preprocessed external data to match our 64-channel locations using the EEGLAB function pop_interp.

After channel interpolation, both internal and external EEG data were analyzed identically. The analysis was applied to individual successful and failed Stop trial epochs $(-500$ to $+2000 \mathrm{~ms}$ with respect to the Stop signal) using custom scripts in MATLAB 2017b and 2018b (MathWorks). After preprocessing, EEG data were transformed using the current source density method (CSD; Kayser and Tenke, 2006; https://psychophysiology.cpmc.columbia. edu/software/CSDtoolbox/index.html) which is a reference-free montage to attenuate the effect of volume conduction in scalp EEG.

\section{Time-frequency transformation}

For all epochs, two-dimensional representations of each electrode's time frequency were estimated using a complex Morlet wavelet (range of logarithmically spaced $4-10$ cycles for 15 linearly spaced frequencies across $15-29 \mathrm{~Hz}$ ). The squared magnitude of the convolved data was calculated to obtain power estimates. $\beta$-bursts were extracted from time-frequency power estimates without baseline normalization. Baseline normalization was separately performed to compute averaged normalized $\beta$-power. Time-frequency power estimates were converted to decibel $(\mathrm{dB})$ using a 100 -ms baseline before the Stop signal. $\beta$-power was estimated over whole epochs and was only later subdivided into smaller time bins. It therefore does not matter if time bin sizes are smaller than the $\beta$-cycle length.

\section{$\beta$-burst detection}

$\beta$-burst detection was performed using the same general method as described in Wessel (2020), originally reported in Shin et al. (2017). For each time-frequency power matrix, local maxima were detected using the MATLAB function imregionalmax, from -25 to $+1000 \mathrm{~ms}$ with respect to the Stop signal. The imregionalmax function identifies data with higher values that are surrounded by data with lower values (starting at $-25 \mathrm{~ms}$ is necessary to inspect lower values for maxima located close to $0 \mathrm{~ms}$ ). $\beta$-bursts were then defined as local maxima that exceeded a defined threshold. While Wessel (2020) and Shin et al. (2017) employed a $\beta$-burst detection threshold of $6 \times$ median power calculated from -500 to $+1000 \mathrm{~ms}$ with respect to the Stop signal (across all trials per subject), we used machine learning to search across six different thresholds ranging from $1 \times$ to $6 \times$ median power of each individual time-frequency power matrix. We also tested two different baselines to calculate median power from, either using the whole epoch including stopping ( -500 to $+1000 \mathrm{~ms}$ with respect to the Stop signal) or using $300 \mathrm{~ms}$ during the fixation cross period. The purpose of this was to establish an optimal burst detection method for detecting $\beta$-bursts in human EEG that yielded the highest predictive ability for the behavior under investigation (the $6 \times$ median power threshold employed by Shin et al. (2017) was decided following a search procedure through a range of thresholds based on prestimulus $\beta$-power derived from murine local field potentials and human magnetoencephalography).

\section{$\beta$-features extraction}

The time-frequency power matrices of each participant were epoched from -125 to $+100 \mathrm{~ms}$ with respect to each participant's SSRT, calculated for each of the 64 scalp electrodes. For each individual 225-ms time-frequency power matrix, features were individually extracted for either 25-, 45-, or 75-ms time bins (i.e., nine, five, or three bins, respectively). Three types of $\beta$-band activity features were extracted in each time bin (Fig. 1): (1) $\beta$-burst rate, the sum of the number of suprathreshold bursts; (2) $\beta$-burst volume, the area under the curve of suprathreshold datapoints, individually calculated for each frequency and subsequently summed up over all frequencies within each time bin to obtain volume ( $\beta$-burst volume was calculated per time bin, and not per burst, so that each trial would have the same number of features for inclusion in the regression models); (3) normalized $\beta$-power, the mean of the baseline normalized power estimates.

\section{Data preparation for the machine learning analysis}

Our internal dataset $(n=218)$ was divided into two groups: the internal validation set $(n=130)$ and the holdout validation set $(n=88)$. In order to balance the internal and holdout validation sets with respect to gender, participants were assigned to each set randomly but the assignment was iterated until the female:male ratio fell within the range 1.545:1$1.655: 1$ in each set. Data from Wessel (2020) served as the external validation set $(n=201)$.

The goal of the analysis was to predict individual trial outcomes (successful or failed stopping) and, separately, individual SSRTs from EEG data. Thus, each trial was regarded as an independent observation. This resulted in 2546 successful Stop trials and 2087 failed Stop trials for the internal validation set, 1814 successful Stop trials and 1454 failed Stop trials for the holdout validation set and 7809 successful Stop trials and 6449 failed Stop trials for the external validation set. For each trial, we obtained a value for each electrode and time bin for each of the three $\beta$-features as described in $\beta$-features extraction.

\section{Experimental design and statistical analysis}

Behavioral analysis

In addition to the SSRT analysis described earlier, means and SDs were extracted for each subject for the following behavioral SST measures: Go trial RT, failed Stop trial RT, SSD, number of successful Stop trials, number of failed Stop trials, probability of successful stopping, probability of Go omissions, probability of choice errors. These measures were compared between the internal and the holdout as well as the external validation sets using a two-sample $t$ test. Go trial RT and failed Stop trial RT were compared within each validation set using a paired $t$ test and effect sizes were estimated using Cohen's $d$. Participants were excluded from analysis if their failed Stop trial RT was larger than Go trial RT (Bissett et al., 2019; Verbruggen et al., 2019), violating an assumption of the horserace model.

\section{Machine learning analysis of the internal validation set}

The detailed method for the machine learning used in this study is described in Rueda-Delgado et al. (2021) and is briefly explained here. We used logistic regression to classify successful versus failed Stop trials (Stop trial classification) and, separately, linear regression to predict individual SSRT for each trial (SSRT prediction). For both analyses we used a form of penalized regression (specifically, the Elastic Net; Zou and Hastie, 2005) to attenuate overfitting (Jollans et al., 2019). The Elastic Net constrains the size of regression coefficients and can also set regression coefficients to zero (i.e., it is a form of feature selection). A modification for this study was that the range of the two hyperparameters $(\alpha$ and $\lambda$ ) were expanded, and the search grid altered. For logistic regression, $\alpha$ ranged from 0.01 to 1 across 10 logarithmically-spaced values and $\lambda$ ranged between 0.2 and 1 across 10 linearly-spaced values 
A Single trial time-frequency spectrum

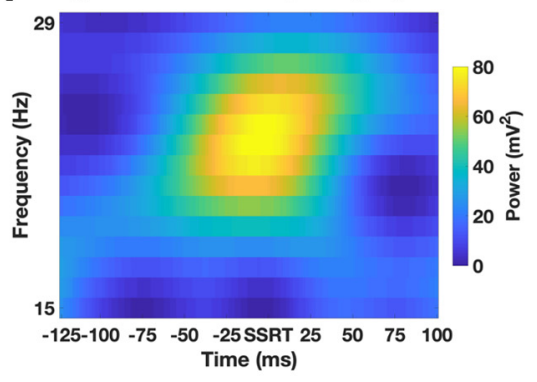

B
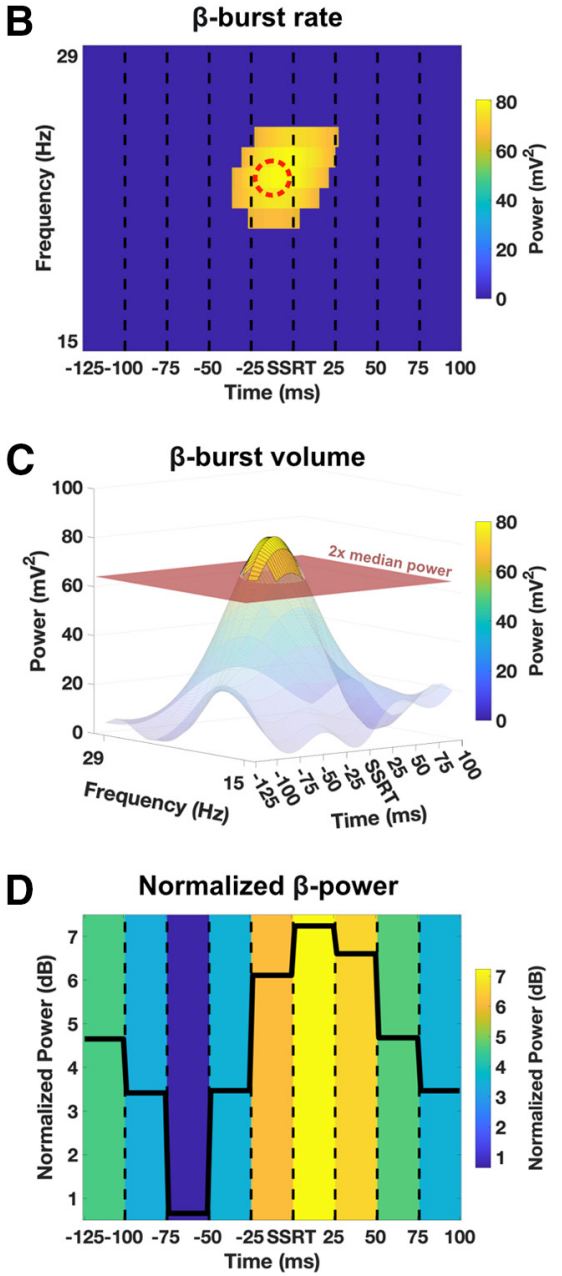

Figure 1. $\quad \beta$-feature extraction from a representative single trial. $\boldsymbol{A}$, Each trial consisted of a 15 (frequencies) $\times 117$ (time points; $225 \mathrm{~ms}, 512 \mathrm{~Hz}$ ) time-frequency power matrix. The 117 time points were divided into nine 25 -ms time bins, from -125 ms before SSRT to $+100 \mathrm{~ms}$ after SSRT. $\beta$-bursts were defined as local maxima which exceeded a predefined threshold. $\boldsymbol{B}, \beta$-burst rate. Shown are all datapoints exceeding the threshold $(2 \times$ median power). Each local maximum (indicated with red dashed circle) exceeding the threshold was counted as one $\beta$-burst for the time bin where it occurred. $\boldsymbol{C}, \beta$-burst volume. All timepoints exceeding the threshold $(2 \times$ median power) were included for the volume calculation. The volume was spread across the different time bins and frequencies. $\boldsymbol{D}$, Normalized $\beta$-power. The normalized power values over all frequencies were averaged for each time bin (means per time bin are indicated with black line).

(i.e., a search grid of 100 parameter-pair values). For linear regression, $\alpha$ ranged from 0.3 to 1 in 10 linearly-spaced values and $\lambda$ ranged from 3 to 7 in 10 linearly-spaced values. Model hyperparameters were determined using nested cross-validation. In contrast to Rueda-Delgado et al. (2021), here, we used 5-fold rather than 10-fold cross-validation. The main folds and subfolds were assigned across subjects. In each subfold, the data were $z$ scored and then Winsorized (i.e., values $>|3|$ were replaced with a value of \pm 3 ). The entire analysis was iterated 100 times for each model, with a new random assignment of training and test sets on each iteration.

We adjusted for age and sex by adding them as additional features to each model (Rubia et al., 2013; Hsieh and Lin, 2017). For the internal and holdout validation sets we additionally controlled for the four different data collection projects by adding three dummy-coded regressors.

To quantify the model performance, the entire procedure was repeated using random-label permutation (i.e., to generate a "null" model) where trial outcomes were randomly shuffled (i.e., the successful or failed Stop trial label for the logistic regression, or SSRT for the linear regression). Note that covariates were not shuffled for null models, thereby quantifying their contribution to the actual model (Dinga et al., 2020). That is, any increase in performance between the actual and null models can be attributed to the contribution of the EEG data. The performance metrics for the logistic regression consisted of the area under the receiver operating characteristic curve (AROC; chance $=0.5$ ) and Brier score (i.e., mean squared error between predicted probabilities and expected values; Dinga et al., 2019). The performance metrics for the linear regression consisted of mean absolute error (MAE) and coefficient of determination ( $R^{2}$; Poldrack et al., 2020). Results reported for the internal validation are mean values across all 100 iterations of the analysis. To assess the significance of the actual model predictions we calculated $P$ which is equal to the fraction of iterations on which the performance metrics of the null model were more predictive than the performance metrics of the actual model (compare Greene et al., 2018).

We sought to first determine the optimal $\beta$-burst detection method by using the internal validation dataset. For this, we tested two parameters: $(1)$ six different thresholds $(1 \times, 2 \times, 3 \times, 4 \times, 5 \times$, or $6 \times$ median power) and (2) two different baselines to calculate median power of, either using the whole epoch including stopping $(-500$ to $+1000 \mathrm{~ms}$ with respect to the Stop signal) or using $300 \mathrm{~ms}$ during the fixation cross period. In addition to these burst detection parameters, we also tested a third parameter: (3) three different time bin sizes to partition the 225-ms time-frequency power matrix $(9 \times 25,5 \times 45$, or $3 \times 75 \mathrm{~ms})$. We tested all 36 possible combinations of these three parameters using a "burst features" model that consisted of $\beta$-burst rate and $\beta$-burst volume. Depending on the time bin size, this model consisted of 1152,640 , or 384 spatiotemporal features (two burst features $\times 64$ channels $\times$ nine, five, or three time bins). The analysis was iterated 100 times for each combination. We ran this analysis both for the Stop trial classification and for the SSRT prediction. We then determined the most predictive parameter combination across both analyses.

Following determination of the optimal $\beta$-burst detection method and time bin size, the internal validation analysis was tested on five final main models using the most predictive parameter combination (see Results): (1) full features model ( $\beta$-burst rate, $\beta$-burst volume, normalized $\beta$-power; 1728 spatiotemporal features); (2) burst features model ( $\beta$-burst rate, $\beta$-burst volume; 1152 spatiotemporal features); and three single feature models: (3) $\beta$-burst rate (576 spatiotemporal features); (4) $\beta$-burst volume (576 spatiotemporal features); and (5) normalized $\beta$-power (576 spatiotemporal features). The performance metrics of the five main models were compared using a one-way repeated measures ANOVA test (omnibus $p$ threshold $=0.05$ ) and a post hoc $t$ test with Bonferroni correction to determine whether the performance metric means of the models were significantly different. Effect sizes were estimated using $\eta^{2}$.

\section{Validation analysis of the holdout and external validation sets}

The results of the five main models from the internal validation were tested on the holdout and external validation sets. This involved applying the optimum burst detection parameters and time bin size as well as regression weights from the internal set to the external and holdout sets (neither the optimum burst detection parameters and time bin size nor the regression weights could be derived from the holdout and external sets as this would violate the independence between training and test sets; Poldrack et al., 2020). The holdout and external validation sets were scaled to the internal validation data by $z$ scoring and Winsorizing using 
Table 1. Electrode labels (64 channels, $10-5$ system) grouped into eight different brain regions

\begin{tabular}{|c|c|}
\hline Brain area & Electrode labels \\
\hline Left parietal & CP5, TP7, P3, P5, P7, P9, P03, P07 \\
\hline Left sensorimotor & $\mathrm{FC} 3, \mathrm{~T} 7, \mathrm{C} 5, \mathrm{C} 3, \mathrm{C} 1, \mathrm{CZ}, \mathrm{CP} 1, \mathrm{CP} 3$ \\
\hline Left frontal & $\mathrm{FP1}, \mathrm{AF7}, \mathrm{AF3}, \mathrm{F7}, \mathrm{F5}, \mathrm{F3}, \mathrm{FT7}, \mathrm{FC5}$ \\
\hline Frontocentral & $\mathrm{FPZ}, \mathrm{AFZ}, \mathrm{FZ}, \mathrm{F1}, \mathrm{F2}, \mathrm{FC} 1, \mathrm{FC}$, FCZ \\
\hline Centroparietal/occipital & PZ, P1, P2, P0Z, 0Z, 01, 02, IZ \\
\hline Right frontal & FP2, AF8, AF4, F8, F6, F4, FT8, FC6 \\
\hline Right sensorimotor & $\mathrm{FC} 4, \mathrm{~T} 7, \mathrm{C} 6, \mathrm{C} 4, \mathrm{C} 2, \mathrm{CPZ}, \mathrm{CP} 2, \mathrm{CP} 4$ \\
\hline Right parietal & CP6, TP8, P4, P6, P8, P10, P04, P08 \\
\hline
\end{tabular}

This step reduced spatial features to aid data interpretation.

the mean and SD of the internal validation set. The mean SSRTs of the holdout and external validation sets were 5 and $51 \mathrm{~ms}$ longer, respectively. Therefore, for the SSRT prediction, the SSRTs in the holdout and external validation sets were scaled to the same mean and SD of the internal validation set SSRT. For each model, the average regression coefficients from the 100 iterations of the internal validation were then applied to the holdout and external validation data. The same metrics to evaluate model performance were utilized for holdout and external validation sets. This procedure was again repeated using random-label permutation (null model).

\section{Data interpretation}

To further interrogate the data and identify the most predictive features in successful models, we calculated the "selection frequency" of each individual feature. This was calculated by summing each feature's nonzero count in each main fold and subsequently averaging across the 100 iterations. Features were then ranked first by selection frequency and second by the absolute regression coefficient. Solely to aid interpretation, we applied spatial and temporal feature reduction to summarize the results. We created boxplots by grouping data from 64 electrodes into 8 different regions (Table 1) and amalgamated the data into three time bins $(-125$ to $-50 \mathrm{~ms}$ before SSRT, -50 to $+25 \mathrm{~ms}$ around SSRT, +25 to $+100 \mathrm{~ms}$ after SSRT).

\section{Code accessibility}

Custom written scripts can be downloaded on the Open Science Framework at https://osf.io/4tznd/.

\section{Results}

\section{Behavioral results}

The behavioral data and statistical comparisons of all three validation sets are displayed in Table 2. Failed Stop RT was larger than Go RT for two participants in the internal validation set, zero participants in the holdout validation set, and two participants in the external validation set. These data were therefore excluded from further analysis. Within each validation set, failed Stop RTs were significantly faster than Go RTs (internal: $t_{(129)}=28.51, p=1.6 \times 10^{-57}, d=2.50$; holdout: $t_{(87)}=23.74$, $p=9.0 \times 10^{-40}, d=2.53$; external: $t_{(200)}=33.54, p=4.6 \times 10^{-84}$, $d=2.37$ ), in accordance with the horse-race model assumption. There were no significant differences in the behavioral measures between internal and holdout validation sets. There were significant differences between every behavioral SST measure except the SSD when comparing internal and external validation sets, which used slightly different versions of the SST.

\section{Machine learning results}

\section{Optimal $\beta$-burst detection method and time bin size}

The results of the analyses to determine the optimal $\beta$-burst detection method and optimal time bin size are displayed in Figure 2. The differences in performance metrics across the 36 models were small. Both the Stop trial classification and the SSRT prediction yielded most predictive results using $1 \times$ median power as the burst detection threshold. However, after visual inspection of the data it was apparent that thresholds below $2 \times$ median power occasionally misregistered background $\beta$-oscillatory activity as bursts. For interpretability (i.e., to distinguish between averaged $\beta$-power and $\beta$-burst events), therefore, we used $2 \times$ median power as the burst detection threshold for both Stop trial classification and SSRT prediction. Further, there was no difference between using either baseline method to calculate median power. This was further supported when correlating the data coming from either baseline which showed that these data correlated (for $\beta$-burst rate: $r=0.83$, for $\beta$-burst volume: $r=0.99$ ). Therefore, we chose -500 to $+1000 \mathrm{~ms}$ as a baseline for compatibility with previous studies (Shin et al., 2017; Wessel, 2020). Finally, there was little difference among different time bin sizes. We therefore chose the 25-ms time bin size because it afforded greater temporal precision.

\section{Stop trial classification (logistic regression results of five main models)}

Figure $3 A$ displays the results for the Stop trial classification for all five main models plus corresponding null models for internal, holdout, and external validation sets.

We aimed to find the features that best classified successful versus failed Stop trials. The mean AROCs of the five models were unequal according to a one-way repeated measures $\operatorname{ANOVA}\left(F_{(4,396)}=1185, p=8.1 \times 10^{-219}, \eta^{2}=0.92\right)$. Post hoc comparisons (Bonferroni corrected at $0.05 / 10=0.005$ ) indicated that mean AROC of the single feature $\beta$-burst volume model (mean AROC $=0.57$, mean Brier score $=0.244$ ) was significantly larger than mean AROC of the full features model (mean $\mathrm{AROC}=0.56$, mean Brier score $=0.245$ ) and of the burst features model (mean AROC $=0.56$, mean Brier score $=0.246$ ). The full features model and the burst features model were the only two models which were not significantly different from each other. The single feature $\beta$-burst volume model outperformed the null model on every iteration $\left(\mathrm{P}_{\mathrm{AROC}}=0, \mathrm{P}_{\mathrm{Brier}}=0\right)$. The holdout (mean $\mathrm{AROC}=0.58$, mean Brier score $=0.243$ ) and the external (mean $\mathrm{AROC}=0.57$, mean Brier score $=0.244$ ) validations yielded similar results when applying the regression coefficients from the internal validation single feature $\beta$-burst volume model. As a next step, we compared the mean AROCs of the three single feature models and the post hoc test revealed that they were all significantly different from each other. The $\beta$-burst rate model showed the worst classification accuracy (mean $\mathrm{AROC}=0.51$, mean Brier score $=0.248$ ) although generally outperformed the null model $\left(\mathrm{P}_{\mathrm{AROC}}=0.24, \mathrm{P}_{\mathrm{Brier}}=0.25\right)$. The null model results of the Stop trial classification (AROCs around 0.5, i.e., chance level) indicate that the model accuracies did not depend on the inclusion of the covariates.

\section{SSRT prediction (linear regression results of five main models)}

Figure $3 B$ displays the results for the SSRT prediction for all five main models plus corresponding null models for internal, holdout, and external validation sets.

The mean MAEs of the five models were unequal according to a one-way repeated measures $\operatorname{ANOVA}\left(F_{(4,396)}=36.23, p=8.3\right.$ $\left.\times 10^{-26}, \eta^{2}=0.27\right)$. Post hoc comparisons (Bonferroni corrected at $0.05 / 10=0.005)$ indicated that mean MAEs of the full features model (mean $\mathrm{MAE}=28.77$, mean $R^{2}=0.02$ ), the burst features model (mean MAE $=28.78$, mean $R^{2}=0.02$ ) and the single feature $\beta$-burst volume model (mean $\mathrm{MAE}=28.82$, mean $R^{2}=$ 
Table 2. Characteristics and statistical comparison of the internal, holdout, and external validation sets

\begin{tabular}{|c|c|c|c|c|c|c|c|}
\hline & \multirow[b]{2}{*}{$\begin{array}{l}\text { Internal } \\
\text { validation set }\end{array}$} & \multirow[b]{2}{*}{$\begin{array}{l}\text { Holdout } \\
\text { validation set }\end{array}$} & \multirow[b]{2}{*}{$\begin{array}{l}\text { External } \\
\text { validation set }\end{array}$} & \multicolumn{2}{|l|}{ Internal-holdout } & \multicolumn{2}{|l|}{ Internal-external } \\
\hline & & & & $\begin{array}{l}\text { Statistical } \\
\text { test (df) }\end{array}$ & $p$ value & $\begin{array}{l}\text { Statistical } \\
\text { test (df) }\end{array}$ & $p$ value \\
\hline Sex (female:male) & $78: 52$ & $55: 33$ & $120: 81$ & $\chi^{2}(1)=0.14$ & 0.71 & $\chi^{2}(1)=0.01$ & 0.96 \\
\hline Age (years) & $35.39(14.41)$ & $33.78(15.35)$ & $22.70(6.83)$ & $t_{(216)}=0.79$ & 0.43 & $t_{(329)}=10.76$ & $2.44 \times 10^{-23}$ \\
\hline SSRT (ms) & $190(36)$ & $195(34)$ & $241(49)$ & $t_{(216)}=-1.13$ & 0.26 & $t_{(329)}=-10.31$ & $8.55 \times 10^{-22}$ \\
\hline SSD (ms) & $278(61)$ & $266(66)$ & $284(123)$ & $t_{(216)}=1.35$ & 0.18 & $t_{(329)}=-0.57$ & 0.57 \\
\hline Mean Go RT (ms) & $486(62)$ & $479(71)$ & $535(105)$ & $t_{(216)}=0.85$ & 0.40 & $t_{(329)}=-4.79$ & $2.55 \times 10^{-6}$ \\
\hline Mean failed Stop RT (ms) & $421(58)$ & $409(66)$ & $460(92)$ & $t_{(216)}=1.42$ & 0.16 & $t_{(329)}=-4.25$ & $2.79 \times 10^{-5}$ \\
\hline Number of successful Stop epochs & $22.68(3.49)$ & $22.78(3.69)$ & $38.85(4.46)$ & $t_{(216)}=-0.22$ & 0.83 & $t_{(329)}=-35.00$ & $5.98 \times 10^{-113}$ \\
\hline Number of failed Stop epochs & $19.63(3.70)$ & $20.16(3.95)$ & $32.09(5.11)$ & $t_{(216)}=-1.01$ & 0.32 & $t_{(329)}=-24.02$ & $2.30 \times 10^{-74}$ \\
\hline Probability of successful Stop & $0.54(0.07)$ & $0.53(0.09)$ & $0.52(0.03)$ & $t_{(216)}=0.51$ & 0.61 & $t_{(329)}=3.17$ & 0.002 \\
\hline Probability of Go omission & $2.49(3.53)$ & $2.27(4.16)$ & $0.03(0.04)$ & $t_{(216)}=0.43$ & 0.67 & $t_{(329)}=9.93$ & $1.65 \times 10^{-20}$ \\
\hline Probability of choice errors & $2.33(2.00)$ & $2.57(3.37)$ & $0.01(0.02)$ & $t_{(216)}=-0.67$ & 0.50 & $t_{(329)}=16.46$ & $8.20 \times 10^{-45}$ \\
\hline
\end{tabular}

For all analyses, $t$ tests were used, except for sex comparisons, for which we used a $\chi^{2}$ test. Means and SDs are reported, except for sex. df, degrees of freedom.

$0.01)$ were not significantly different, however, they were significantly more predictive than the single feature models $\beta$-burst rate and normalized $\beta$-power. There was a modest performance improvement in comparison to the null model: full features model $\left(\mathrm{P}_{\mathrm{MAE}}=0.27, \mathrm{P}_{\mathrm{R} 2}=0.28\right)$, burst features model $\left(\mathrm{P}_{\mathrm{MAE}}=\right.$ $\left.0.29, \mathrm{P}_{\mathrm{R} 2}=0.23\right)$ and single feature $\beta$-burst volume model $\left(\mathrm{P}_{\mathrm{MAE}}=0.34, \mathrm{P}_{\mathrm{R} 2}=0.36\right)$. The respective holdout and the external validations yielded similar results. The mean MAEs of the two remaining single features models, $\beta$-burst rate (mean MAE $=29.18$, mean $R^{2}=0.00$ ) and normalized $\beta$-power (mean $\mathrm{MAE}=29.17$, mean $R^{2}=0.01$ ), were not significantly different. Neither model outperformed the null models ( $\beta$-burst rate: $\left.\mathrm{P}_{\mathrm{MAE}}=0.52, \mathrm{P}_{\mathrm{R} 2}=0.54\right)$; normalized $\beta$-power: $\mathrm{P}_{\mathrm{MAE}}=0.49, \mathrm{P}_{\mathrm{R} 2}$ $=0.49)$. The coefficients of determination $\left(R^{2}\right)$ of the null models of the SSRT prediction are slightly above zero, showing an additional, but quite small, effect of the covariates on the SSRT prediction.

\section{Spatial and temporal dynamics of $\boldsymbol{\beta}$-burst volume}

The $\beta$-burst volume model could predict Stop trial classification. We therefore present the spatial and temporal dynamics for this model in more detail. The models did not robustly predict SSRT and therefore we do not interpret their detailed spatial and temporal dynamics here.

\section{Stop trial classification}

Figure 4 shows the spatial and temporal dynamics of $\beta$-burst volume for classifying successful versus failed Stop trials. Larger $\beta$-burst volume in right frontal, frontocentral and bilateral sensorimotor was predictive of successful stopping -125 to $-50 \mathrm{~ms}$ before SSRT. In centroparietal and occipital sites smaller $\beta$-burst volume was predictive of successful stopping (from the other perspective; larger $\beta$-burst volume was predictive of failed stopping). A similar pattern was observed from -50 to $+25 \mathrm{~ms}$ around the SSRT where smaller $\beta$-burst volume in centroparietal and occipital sites was predictive of successful stopping. After the SSRT ( +25 to $+100 \mathrm{~ms}$ ), larger $\beta$-burst volume in bilateral sensorimotor areas was predictive of successful stopping.

\section{Spatial and temporal dynamics of response inhibition across validation sets}

Figure 5 presents topoplots of successful minus failed Stop trials. The three main rows show the data for each $\beta$-feature (i.e., burst rate, burst volume and normalized power). Topoplots are shown for each validation set (i.e., internal, holdout, and external) separately in sub-rows. The pattern of activation across time for $\beta$-burst volume and normalized $\beta$-power is broadly similar across all three validation sets. $\beta$-burst rate is less consistent across validation sets because of the low frequency of burst detection.

\section{Discussion}

Here, we investigated the role of $\beta$-bursts for successful response inhibition and their impact on the speed of stopping (SSRT). We used machine learning to search across a wide span of temporal and spatial features on a large dataset, and employed best practice for validation, including external validation. Results partially supported our hypothesis. $\beta$-burst volume, but not rate, was the superior predictor for Stop trial classification. However, normalized $\beta$-power was a better predictor than $\beta$-burst rate. Stop trial classification (success vs fail) generalized to an external dataset, but prediction of individual SSRT did not.

To the best of our knowledge, this study is the first to introduce $\beta$-burst volume as a key behaviorally relevant measure of human action stopping. Burst volume is a composite measure, capturing in single metric features previously studied in isolation: burst peak amplitude (Feingold et al., 2015; Sherman et al., 2016; Shin et al., 2017; Tinkhauser et al., 2017; Little et al., 2019), burst duration (Feingold et al., 2015; Sherman et al., 2016; Shin et al., 2017; Tinkhauser et al., 2017; Little et al., 2019), and burst frequency span (Shin et al., 2017). Burst amplitude increases in proportion with burst duration (Tinkhauser et al., 2017), suggesting a single generating mechanism and therefore suitable to be combined. The inclusion of a third dimension (frequency span) allows a compact assessment across the entire set of $\beta$-range frequencies (Zich et al., 2020). The $\beta$-rhythm likely inhibits neural processing (Sherman et al., 2016) and the brevity of $\beta$-bursts may be critical for intact messaging in the brain (Feingold et al., 2015). In patients with Parkinson's disease shorter $\beta$-burst duration was related to improved motor function (Tinkhauser et al., 2017). Taken together, these findings suggest burst volume better captures the multifaceted nature of the $\beta$-bursts rather than merely their presence or rate.

Machine learning facilitates a data-driven approach, searching a large area of scalp and wide temporal window. Increased right frontal $\beta$-burst volume from -100 to $-50 \mathrm{~ms}$ before SSRT was predictive of successful stopping. This is spatially and temporally consistent with $\beta$-rhythm findings using electrocorticography (ECoG; Swann et al., 2009, 2012), EEG (Wagner et al., 
A

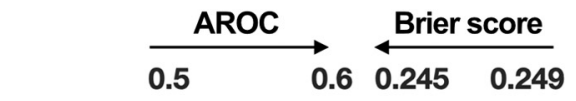

I $*$ *

T1_Full_25 * $\longrightarrow$

T1_Fix_45*

T2_Full_25 * $\quad \mathrm{D}$

T1_Full_45 *

T2_Fix_25 *

T2_Full_75 * T

T3_Fix_45* $>$

T3_Full_25 * $>$

T2_Full_45 * -

T5_Fix_75 * -

T1_Fix_75* $\mathrm{D}$

T6_Fix_25 $*-\mathrm{C}$

T3_Fix_75 * -

T3_Full_45 $*-\mathrm{D}$

T2_Fix_45 * $\quad$ D

T4_Fix_45 * $\longrightarrow$

T3_Fix_25 * D

T4_Fix_25* T

T6_Fix_45* D

T5_Fix_25 * D

T1_Full_75 * D

T4_Full_25 * $<$

T6_Full_45 * >

T4_Fix_75 * D

T2_Fix_75 * D

T3_Full_75 * D

T6_Fix_75* - I

T5_Fix_45 * D

T6_Full_25 * D

T4_Full_45 $*$ D

T5_Full_75*

T4_Full_75 * $\quad \mathrm{C}$

T6_Full_75 $*$ D

T5_Full_25*

T5_Full_45* $\quad$ C

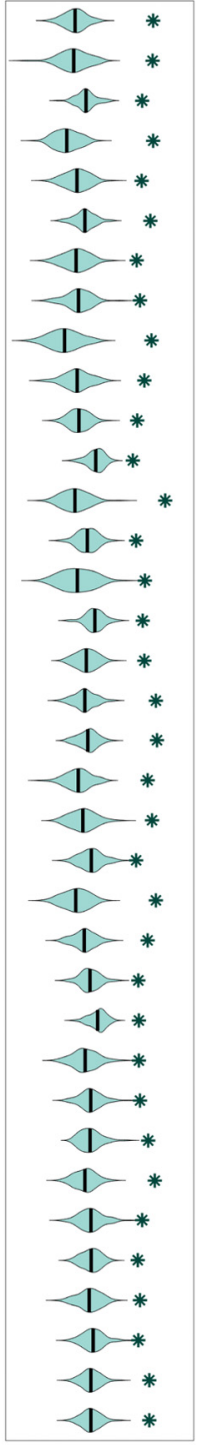

B

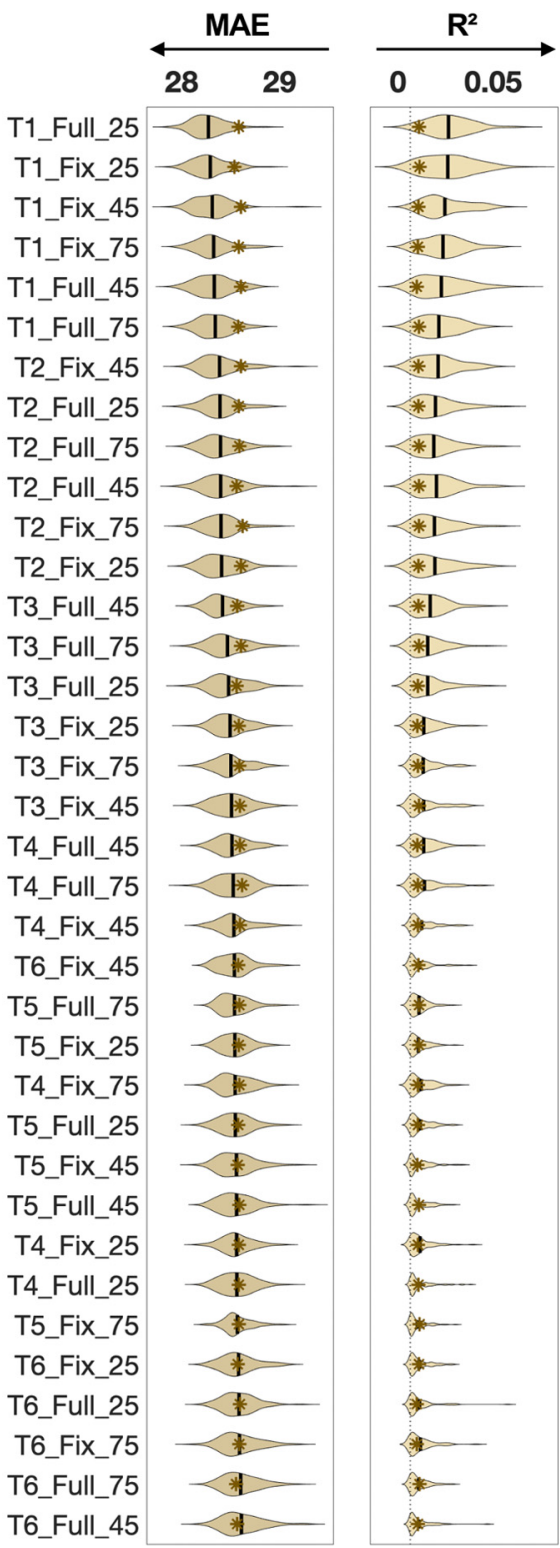

Legend for model names:

Burst detection threshold:

'T1' = 1x median power threshold (etc. for 1-6x median power)

Baseline to calculate median power: 'Full' $=-500 \mathrm{~ms}$ to $+1000 \mathrm{~ms}$ with respect to Stop signal

'Fix' = $300 \mathrm{~ms}$ during fixation cross

Time bin size:

'25' = $25 \mathrm{~ms}$; '45' = $45 \mathrm{~ms}$; '75' = $75 \mathrm{~ms}$

Figure 2. Machine learning results for Optimal $\beta$-burst detection method and time bin size (see section in Materials and Methods; 36 parameter combination models: burst detection threshold $\times$ baseline for median power calculation $\times$ time bin size). $\boldsymbol{A}$, Logistic regression results over 100 iterations for the Stop trial classification are shown. Two performance metrics are reported: AROC and Brier score. The models are sorted by AROC from most predictive to least predictive. Respective null models of each model are indicated with an asterisk symbol. $\boldsymbol{B}$, Linear regression results over 100 iterations for SSRT prediction are shown. Two performance metrics are reported; MAE and $R^{2}$. The models are sorted by MAE from most predictive to least predictive. Respective null models of each model are indicated with an asterisk symbol. Arrows below each performance metric indicate the direction for higher prediction.

2018; Hannah et al., 2020; Jana et al., 2020), and MEG (Schaum et al., 2020). The rIFC is thought to act as a brake for motor output that can be triggered by a unexpected event (Aron et al., 2014; Schaum et al., 2020). We used scalp EEG, which cannot identify the source generator. However, studies using ECoG (Swann et al., 2009, 2012), MEG/functional magnetic resonance imaging (fMRI; Schaum et al., 2020), and fMRI-guided repetitive transcranial magnetic stimulation (Sundby et al., 2021) have empirically demonstrated the link between right frontal scalp activity and rIFC. The larger $\beta$-burst volume recorded from right frontal scalp therefore likely indicates rIFC-related motor braking triggered by the Stop signal. Additionally, we found that early increased bilateral sensorimotor $\beta$-burst volume from $-125 \mathrm{~ms}$ to the time of SSRT predicted successful stopping. This is consistent with $\beta$-burst studies (Jana et al., 2020; Wessel, 2020) that related this finding to global motor suppression during non-selective movement stopping (Badry et al., 2009; Wessel and Aron, 2013). An unexpected result occurred in most time bins: a relative decrease in $\beta$-burst volume in centroparietal and occipital areas for successful Stop trials, potentially indicating relatively more focused activity in successful stopping. Finally, +75 to $+100 \mathrm{~ms}$ after the SSRT, there was a clear sensorimotor 


\begin{tabular}{|lcl|}
\hline $\begin{array}{l}\text { Internal validation } \\
\text { Internal validation null model }\end{array}$ & $+\begin{array}{l}\text { Holdout validation } \\
\text { Holdout validation null model }\end{array}$ & $\begin{array}{c}\text { External validation } \\
\text { External validation null model }\end{array}$ \\
\hline
\end{tabular}

A
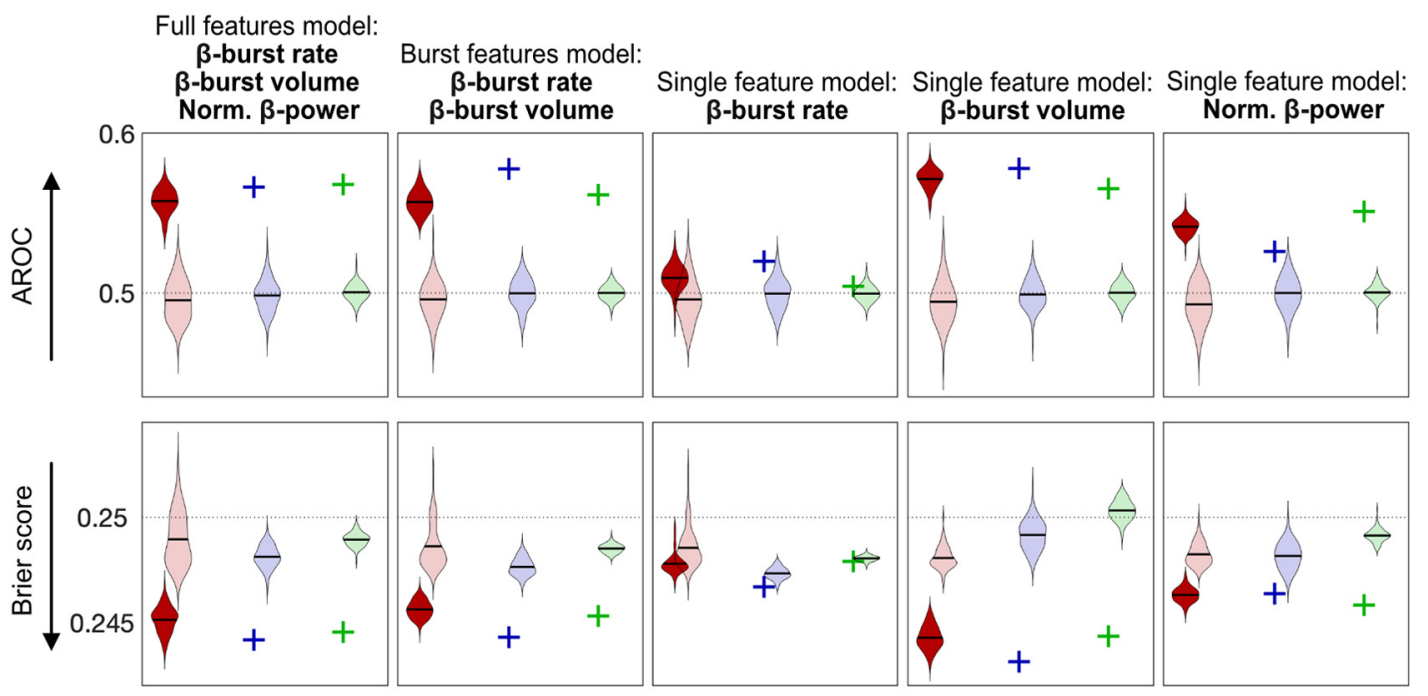

B

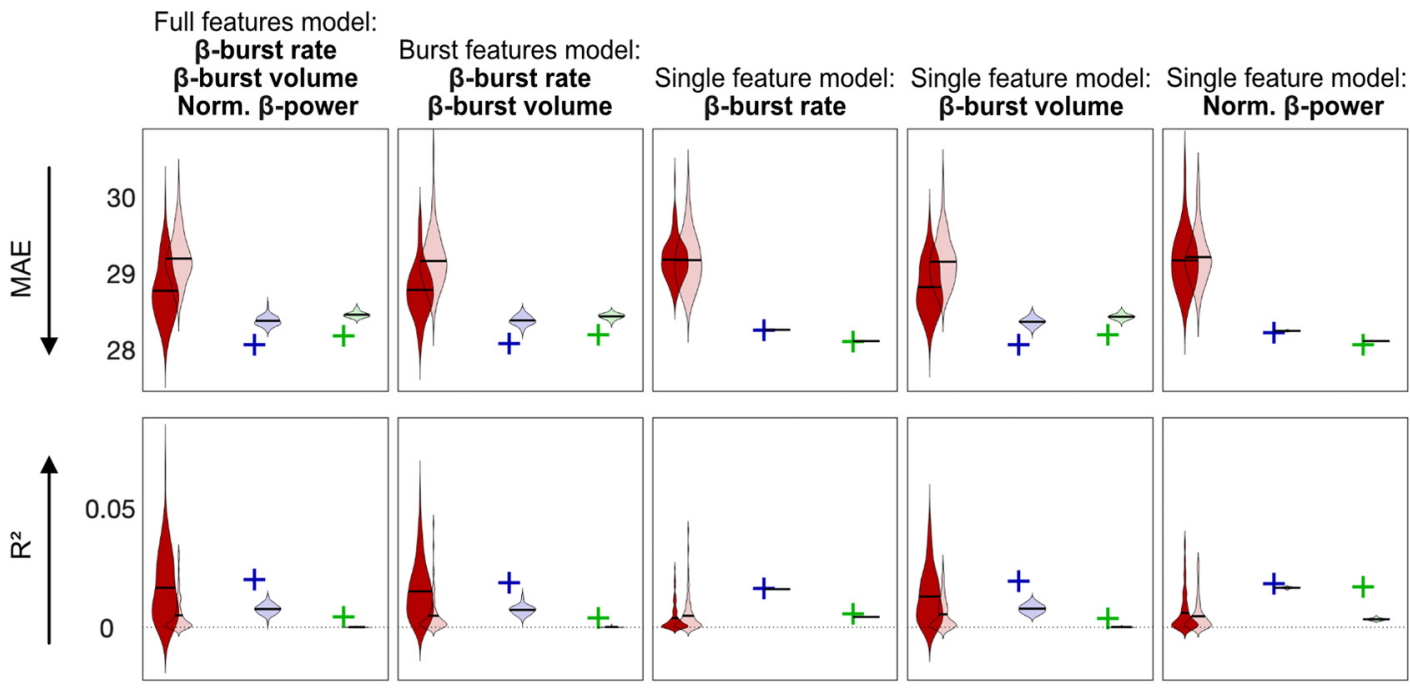

Figure 3. Machine learning results for internal, holdout, and external validation and the corresponding null models of the five main models. $A$, Logistic regression results over 100 iterations for the Stop trial classification are shown. Two performance metrics are reported: AROC and Brier score. $\boldsymbol{B}$, Linear regression results over 100 iterations for the SSRT prediction are shown. Two performance metrics are reported: MAE and $R^{2}$. Dotted lines represent chance level for the respective metric. Arrows beside each performance metric indicate the direction for higher prediction. The holdout and external validations are indicated with a single cross (instead of a distribution over 100 iterations) because mean regression coefficients from the internal validation were applied to the holdout and external data, which resulted in a single performance metric. Norm., normalized.

signature where decreasing $\beta$-burst volume predicted failed stopping, consistent with the well-known $\beta$-desynchronization over motor cortex during motor preparation, which occurs during failed Stop trials where a button press takes place (Zhang et al., 2008; Swann et al., 2009; Fonken et al., 2016).

In contrast to the findings relating to burst volume, burst rate was not a robust predictor of stopping behavior. Similarly, Jana et al. (2020) found no difference in right frontal $\beta$-burst rate between successful versus failed Stop trials, but Wessel (2020; i.e., our external dataset) did at a single frontocentral electrode $(\mathrm{FCz})$. The external dataset SST had a higher Stop trial probability than the internal dataset $(0.33$ vs 0.25$)$. Increased Stop trial probability may recruit a more proactive response inhibition (Castro-Meneses et al., 2015). Some studies suggested that presupplementary motor area (pre-SMA) is more strongly activated during proactive response inhibition (Sharp et al., 2010; Hu et al., 2015), potentially explaining a more frontocentral signature in the external dataset (also compare Leunissen et al., 2020).

Several methods for $\beta$-burst detection amid background brain activity have been described (compare Shin et al., 2017; Tinkhauser et al., 2017; Little et al., 2019; Seedat et al., 2020). There is no consensus on the methodology for optimal $\beta$-burst detection and therefore we tested several thresholds. A threshold of $2 \times$ median $\beta$-power was most sensitive for investigation of the brain's stopping process, suggesting $\beta$-burst information might be lost by using a higher detection threshold. Threshold selection is also relevant to the relationship between burst rate and volume. At higher thresholds, only the larger amplitude bursts will be identified and burst rate will be somewhat confounded with burst volume. Additionally, we tested two different baselines to calculate median power; there was no difference in results and therefore it appears either method can be used for burst threshold calculation. 
A

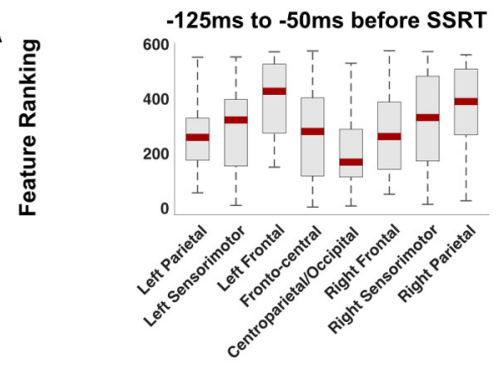

B
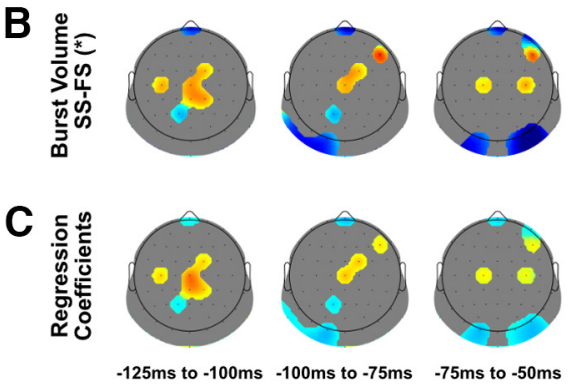
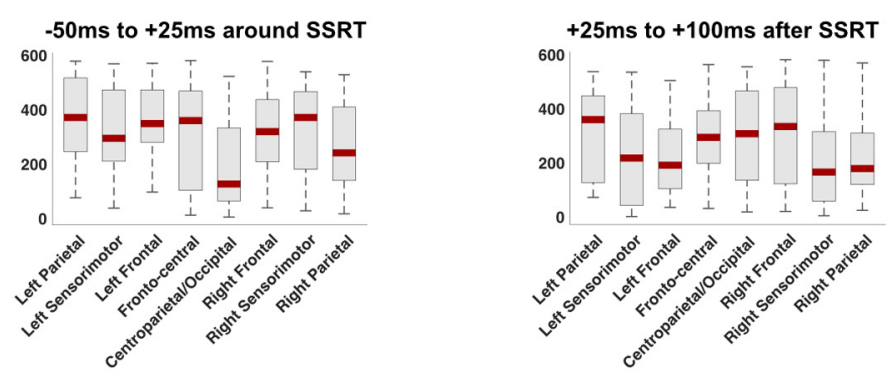

Figure 4. Spatial and temporal $\beta$-burst volume dynamics for the Stop trial classification. $\boldsymbol{A}$, Boxplots display the machine learning feature ranking (ranked after selection frequency and absolute regression coefficient). Low ranking means more predictive in the machine learning analysis. These were averaged over three time bins (75 ms each) and for each time bin averaged over eight different brain regions. $\boldsymbol{B}, \beta$-burst volume data (successful minus failed Stop trials, a.u.) from the top 75 features (over all time bins) are shown for each 25 -ms time bin with the rest of the electrodes masked. C, Regression coefficients from the machine learning logistic regression analysis from the top 75 features (over all time bins) are shown for each 25 -ms bin with the rest of the electrodes masked. SS, successful Stop trial; FS, failed Stop trial. $* \beta$-burst volume is burst duration $\times$ frequency span $\times$ amplitude.

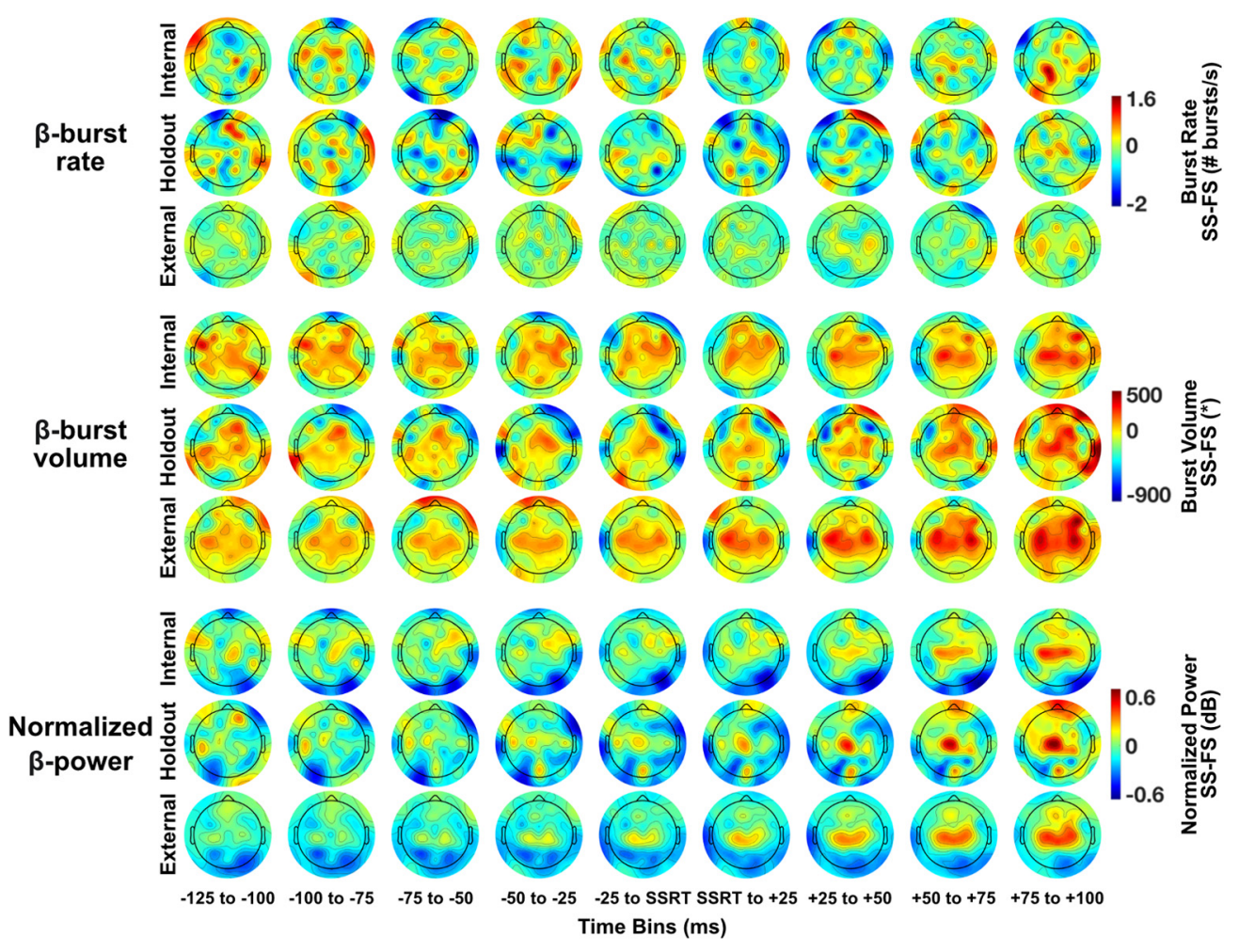

Figure 5. Topoplots of each $\beta$-feature ( $\beta$-burst rate, $\beta$-burst volume, normalized $\beta$-power) for the internal, holdout, and external validation sets. Topoplots are shown for each 25 -ms bin. $\beta$-band frequency range was $15-29 \mathrm{~Hz}$. $\beta$-bursts were calculated using $2 \times$ median power as burst detection threshold. Means are shown for $\beta$-burst rate and normalized $\beta$-power, medians are shown for $\beta$-burst volume. Data are shown for successful minus failed Stop trials for each $\beta$-feature. SS, successful Stop trial; FS, failed Stop trial. $* \beta$-burst volume is burst duration $\times$ frequency span $\times$ amplitude.

Stopping behavior can be described by formal computational models (compare Bissett et al., 2019). For example, the interactive race (Boucher et al., 2007) and the blocked input (Logan et al., 2015) models both characterize Stop and Go processes as stochastic accumulators. For both models, the Stop unit should be active on every successfully inhibited trial (Logan et al., 2015; Errington et al., 2020) to either inhibit or block the Go unit. Despite evidence for $\beta$-involvement in stopping, studies to date 
have not shown that $\beta$-bursts are causally linked as a mechanism for the Stop unit. We consider a number of potential, not mutually exclusive, explanations (see also Errington et al., 2020; Wessel, 2020). First, infrequency of $\beta$-burst detection is because of the low signal-to-noise ratio (SNR) in EEG (Jana et al., 2020). However, Errington and colleagues demonstrated similar frequency of $\beta$-burst detection using intracranial electrodes in macaques, a method with better signal fidelity than EEG. It therefore seems unlikely that poor SNR is the sole reason for the low detection rate. Second, $\beta$-activity is produced by multiple brain sources that reflect different processes: our results clearly support this interpretation. $\beta$-activity topography changed over time, and different scalp regions were predictive of stopping at different times. Third, as suggested by Errington et al. (2020), some informative $\beta$-bursts may be subthreshold. Here, we utilized a training dataset to optimize burst detection threshold, which was lower than previous studies. There was an improvement in prediction accuracy using this lower threshold, but it did not approach the one-to-one mapping necessary to characterize the Stop unit. We suggest that brain activity needs to be recorded with improved SNR (perhaps recording many more trials for EEG). In addition, more sophisticated methods (e.g., deep learning; Abrol et al., 2021) may improve detection of informative bursts and could better integrate information from multiple regions to adequately capture the complexity of $\beta$-band activity in stopping behavior.

The internal validation for the SSRT prediction revealed a weak model that did not generalize to the holdout and external validation data. It is possible that $\beta$-band activity is unimportant for speed of stopping. Another potential explanation is that we relied on the stopping speed derived from a button press to measure SSRT. While this is a widely used measure in response inhibition research, it has its drawbacks (Skippen et al., 2020). Most importantly for our study, the SSRT is only a summary measure for each participant and does not provide single trial information. It is likely that using single trial EMG data to calculate CancelTime (Jana et al., 2020; also compare Thunberg et al., 2020) as the stopping outcome measure would have yielded a more predictive model.

In conclusion, $\beta$-burst volume was superior to both $\beta$-burst rate and averaged normalized $\beta$-power for classifying successful response inhibition. To date, several studies have shown a statistical association between $\beta$-band activity and stopping (Wagner et al., 2018; Jana et al., 2020; Schaum et al., 2020; Wessel, 2020). Here, a machine learning approach reliably showed that $\beta$-bursts are implicated in human action stopping. The models significantly outperformed a random model suggesting a real effect of $\beta$-activity and the classification models were validated on unseen and independent external data. This first validation of neural EEG SST data on a holdout and external dataset therefore fulfils the requirement for establishing prediction (Poldrack et al., 2020). These results support the emerging view that transient $\beta$-bursts are a more accurate representation of oscillatory $\beta$-activity in the brain, and suggest an important role for $\beta$-bursts in human response inhibition.

\section{References}

Abrol A, Fu Z, Salman M, Silva R, Du Y, Plis S, Calhoun V (2021) Deep learning encodes robust discriminative neuroimaging representations to outperform standard machine learning. Nat Commun 12:353.

Aron AR, Robbins TW, Poldrack RA (2014) Inhibition and the right inferior frontal cortex: one decade on. Trends Cogn Sci 18:177-185.
Badry R, Mima T, Aso T, Nakatsuka M, Abe M, Fathi D, Foly N, Nagiub H, Nagamine T, Fukuyama H (2009) Suppression of human cortico-motoneuronal excitability during the stop-signal task. Clin Neurophysiol 120:1717-1723.

Bissett PG, Jones HM, Poldrack RA, Logan GD (2019) Severe violations of independence in response inhibition tasks. Sci Adv 7:eabf 4355.

Boucher L, Palmeri TJ, Logan GD, Schall JD (2007) Inhibitory control in mind and brain: an interactive race model of countermanding saccades. Psychol Rev 114:376-397.

Castro-Meneses LJ, Johnson BW, Sowman PF (2015) The effects of impulsivity and proactive inhibition on reactive inhibition and the go process: insights from vocal and manual stop signal tasks. Front Hum Neurosci 9:529.

Delorme A, Makeig S (2004) EEGLAB: an open source toolbox for analysis of single-trial EEG dynamics including independent component analysis. J Neurosci Methods 134:9-21.

Dinga R, Penninx BWJH, Veltman DJ, Schmaal L, Marquand AF (2019) Beyond accuracy: measures for assessing machine learning models, pitfalls and guidelines. bioRxiv 743138 .

Dinga R, Schmaal L, Penninx BWJH, Veltman DJ, Marquand AF (2020) Controlling for effects of confounding variables on machine learning predictions. bioRxiv 255034 .

Errington SP, Woodman GF, Schall JD (2020) Dissociation of medial frontal $\beta$-bursts and executive control. J Neurosci 40:9272-9282.

Feingold J, Gibson DJ, Depasquale B, Graybiel AM (2015) Bursts of beta oscillation differentiate postperformance activity in the striatum and motor cortex of monkeys performing movement tasks. Proc Natl Acad Sci USA 112:13687-13692.

Fonken YM, Rieger JW, Tzvi E, Crone NE, Chang E, Parvizi J, Knight RT, Krämer UM (2016) Frontal and motor cortex contributions to response inhibition: evidence from electrocorticography. J Neurophysiol 115:2224-2236.

Greene AS, Gao S, Scheinost D, Constable RT (2018) Task-induced brain state manipulation improves prediction of individual traits. Nat Commun 9:2807.

Hannah R, Muralidharan V, Sundby KK, Aron AR (2020) Temporally-precise disruption of prefrontal cortex informed by the timing of beta bursts impairs human action-stopping. Neuroimage 222:117222.

Hsieh S, Lin YC (2017) Stopping ability in younger and older adults: behavioral and event-related potential. Cogn Affect Behav Neurosci 17:348363.

Hu S, Ide JS, Zhang S, Li C-SR (2015) Anticipating conflict: neural correlates of a Bayesian belief and its motor consequence. Neuroimage 119:286295.

Jana S, Hannah R, Muralidharan V, Aron AR (2020) Temporal cascade of frontal, motor and muscle processes underlying human action-stopping. Elife 9:e50371.

Jollans L, Boyle R, Artiges E, Banaschewski T, Desrivières S, Grigis A, Martinot JL, Paus T, Smolka MN, Walter H, Schumann G, Garavan H, Whelan R (2019) Quantifying performance of machine learning methods for neuroimaging data. Neuroimage 199:351-365.

Kayser J, Tenke CE (2006) Principal components analysis of Laplacian waveforms as a generic method for identifying ERP generator patterns: I. Evaluation with auditory oddball tasks. Clin Neurophysiol 117:348-368.

Leunissen I, van Steenkiste M, Heise KF, Monteiro TS, Dunovan K, Mantini D, Coxon JP, Swinnen SP (2020) Effects of beta- and gamma-band rhythmic stimulation on motor inhibition. bioRxiv 422006.

Lijffijt M, Kenemans JL, Verbaten MN, Van Engeland H (2005) A meta-analytic review of stopping performance in attention-deficit/hyperactivity disorder: deficient inhibitory motor control? J Abnorm Psychol 114:216222.

Little S, Bonaiuto J, Barnes G, Bestmann S (2019) Human motor cortical beta bursts relate to movement planning and response errors. PLoS Biol 17: e3000479.

Logan GD, Cowan WB (1984) On the ability to inhibit thought and action: a theory of an act of control. Psychol Rev 91:295-327.

Logan GD, Yamaguchi M, Schall JD, Palmeri TJ (2015) Inhibitory control in mind and brain 2.0: blocked-input models of saccadic countermanding. Psychol Rev 122:115-147.

Luijten M, Littel M, Franken IHA (2011) Deficits in inhibitory control in smokers during a Go/Nogo task: an investigation using event-related brain potentials. PLoS One 6:e18898. 
Nolan H, Whelan R, Reilly RB (2010) FASTER: fully automated statistical thresholding for EEG artifact rejection. J Neurosci Methods 192:152-162.

Poldrack RA, Huckins G, Varoquaux G (2020) Establishment of best practices for evidence for prediction: a review. JAMA Psychiatry 77:534-540.

Richards JB, Zhang L, Mitchell SH, de Wit H (1999) Delay or probability discounting in a model of impulsive behavior: effect of alcohol. J Exp Anal Behav 71:121-143.

Rubia K, Lim L, Ecker C, Halari R, Giampietro V, Simmons A, Brammer M, Smith A (2013) Effects of age and gender on neural networks of motor response inhibition: from adolescence to mid-adulthood. Neuroimage 83:690-703.

Rueda-Delgado LM, Halloran LO, Enz N, Ruddy KL, Kiiski H, Bennett M, Farina F, Jollans L, Vahey N, Whelan R (2021) Brain event-related potentials predict individual differences in inhibitory control. Int $\mathrm{J}$ Psychophysiol 162:22-34.

Schaum M, Pinzuti E, Sebastian A, Lieb K, Fries P, Mobascher A, Jung P, Wibral M, Tüscher O (2020) Cortical network mechanisms of response inhibition. bioRxiv 940841.

Seedat ZA, Quinn AJ, Vidaurre D, Liuzzi L, Gascoyne LE, Hunt BAE, O’Neill GC, Pakenham DO, Mullinger KJ, Morris PG, Woolrich MW, Brookes MJ (2020) The role of transient spectral 'bursts' in functional connectivity: a magnetoencephalography study. Neuroimage 209:116537.

Sharp DJ, Bonnelle V, De Boissezon X, Beckmann CF, James SG, Patel MC, Mehta MA (2010) Distinct frontal systems for response inhibition, attentional capture, and error processing. Proc Natl Acad Sci USA 107:61066111.

Sherman MA, Lee S, Law R, Haegens S, Thorn CA, Hämäläinen MS, Moore CI, Jones SR (2016) Neural mechanisms of transient neocortical beta rhythms: converging evidence from humans, computational modeling, monkeys, and mice. Proc Natl Acad Sci USA 113:E4885-E4894.

Shin H, Law R, Tsutsui S, Moore CI, Jones SR (2017) The rate of transient beta frequency events predicts behavior across tasks and species. Elife 6: e29086.

Skippen P, Fulham WR, Michie PT, Matzke D, Heathcote A, Karayanidis F (2020) Reconsidering electrophysiological markers of response inhibition in light of trigger failures in the stop-signal task. Psychophysiology 57: e13619.

Sundby KK, Jana S, Aron AR (2021) Double-blind disruption of right inferior frontal cortex with TMS reduces right frontal beta power for action stopping. J Neurophysiol 125:140-153.
Swann N, Tandon N, Canolty R, Ellmore TM, McEvoy LK, Dreyer S, DiSano $M$, Aron AR (2009) Intracranial EEG reveals a time- and frequency-specific role for the right inferior frontal gyrus and primary motor cortex in stopping initiated responses. J Neurosci 29:12675-12685.

Swann NC, Cai W, Conner CR, Pieters TA, Claffey MP, George JS, Aron AR, Tandon N (2012) Roles for the pre-supplementary motor area and the right inferior frontal gyrus in stopping action: electrophysiological responses and functional and structural connectivity. Neuroimage 59:2860-2870.

Thunberg C, Messel MS, Raud L, Huster RJ (2020) tDCS over the inferior frontal gyri and visual cortices did not improve response inhibition. Sci Rep 10:7749.

Tinkhauser G, Pogosyan A, Little S, Beudel M, Herz DM, Tan H, Brown P (2017) The modulatory effect of adaptive deep brain stimulation on beta bursts in Parkinson's disease. Brain 140:1053-1067.

Verbruggen F, Aron AR, Band GP, Beste C, Bissett PG, Brockett AT, Brown JW, Chamberlain SR, Chambers CD, Colonius H, Colzato LS, Corneil BD, Coxon JP, Dupuis A, Eagle DM, Garavan H, Greenhouse I, Heathcote A, Huster RJ, Jahfari S, et al. (2019) Capturing the ability to inhibit actions and impulsive behaviors: a consensus guide to the stop-signal task. Elife 8:e46323.

Wagner J, Wessel JR, Ghahremani A, Aron AR (2018) Establishing a right frontal beta signature for stopping action in scalp EEG: implications for testing inhibitory control in other task contexts. J Cogn Neurosci 30:107118.

Wessel JR (2020) $\beta$-Bursts reveal the trial-to-trial dynamics of movement initiation and cancellation. J Neurosci 40:411-423.

Wessel JR, Aron AR (2013) Unexpected events induce motor slowing via a brain mechanism for action-stopping with global suppressive effects. J Neurosci 33:18481-18491.

Wessel JR, Aron AR (2017) On the globality of motor suppression: unexpected events and their influence on behavior and cognition. Neuron 93:259-280.

Zhang Y, Chen Y, Bressler SL, Ding M (2008) Response preparation and inhibition: the role of the cortical sensorimotor beta rhythm. Neuroscience 156:238-246.

Zich C, Quinn AJ, Mardell LC, Ward NS, Bestmann S (2020) Dissecting transient burst events. Trends Cogn Sci 24:784-788.

Zou H, Hastie T (2005) Regularization and variable selection via the elastic net. J R Stat Soc Series B Stat Methodol 67:301-320. 